Peter Canisius

Pécs / Nemesvámos

\title{
Genus (nicht nur) im Ungarischen ${ }^{1}$
}

\author{
DOI: 10.14232/fest.bassola.2
}

\begin{abstract}
Das Ungarische hat, so die herrschende Lehre, kein Genus. Ausgehend von der die Genera des Griechischen an die Artikel bindenden Genuskonzeption des Dionysios Thrax und einem Blick auf die von den pronominalen und adjektivischen Substantivsatelliten bestimmten Genera des Lateinischen und die ausschließlich auf Pronomina rekurrierende Unterscheidung der Genera im Englischen versuche ich zu zeigen, daß und inwiefern die herrschende Lehre vom genuslosen Ungarischen falsch ist.
\end{abstract}

\section{Start und Ziel}

In G. Corbetts „Gender“ kommt „Hungarian“ nur einmal vor, und da wird klargestellt: „Uralic has some members in Europe (like Hungarian) and others in the northern area where Europe and Asia meet, and is devoid of grammatical gender.“ (1991: 2) Auch Ch. J. Beckwith beginnt seinen Aufsatz „Classifiers in Hungarian“ mit einer klaren Aussage:

In both the Uralistic literature $[. .$.$] and the literature on linguistic ty-$ pology $[\ldots]$ it is stated that Uralic languages, in particular Hungarian, do not have gender (Corbett $(1991,2)$ ), that is, grammatical noun class with overtly marked concord. (Beckwith 1992: 197)

Im Register von T. Forgács oder B. Keszlers / K. Lengyels ungarischer Grammatik oder der von Keszler herausgegebenen „Magyar grammatika“ kommt

Dieser Beitrag geht zurück auf einen meiner beiden Habilitationsvorträge. Eines der damaligen Jurymitglieder war jemand, mit dem ich schon während meiner Tätigkeit an der Katholischen Péter-Pázmány-Universität 1994/95 sehr gerne zusammengearbeitet habe: Péter Bassola. 
„Genus (nominis)“ bzw. „nem“ („Geschlecht“) gar nicht vor, und zwar eben deshalb nicht, weil es, wie auch J. Tompa (1985: 147) weiß, „das grammatische Geschlecht [...] im Ung. gar nicht gibt“.

Corbett zitiert in seiner Definition von Genus „Only one type of classification counts as a gender system; it is one which is reflected beyond the nouns themselves in modifications required by ,associated words." (1991: 4) die in der modernen Genusforschung wohl am häufigsten zitierte und zugrunde gelegte Genuskonzeption, die von Ch. F. Hockett: „Genders are classes of nouns reflected in the behavior of associated words“. $(1958: 231)^{2}$

Und auch ich lege diese Hockettsche Konzeption zugrunde. Doch ich komme zu einem anderen Schluss als die herrschende Lehre.

\section{Die Anfänge: Ein Blick ins Griechische}

Vor mehr als 2100 Jahren schreibt Dionysios Thrax (ca. 170 - ca. 90 v. Chr.) in

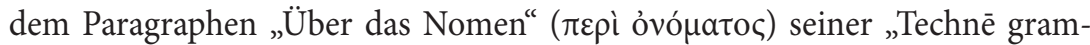

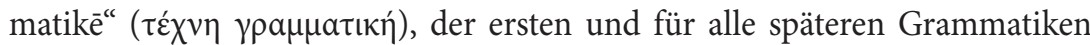
grundlegenden Grammatik, über das Genus: „Genera gibt es drei: Maskulin, feminin und neutrum. ${ }^{\text {"3 }}$

In dem Abschnitt über den Artikel ( $\pi \varepsilon \rho \grave{~} a ̈ \rho \theta \rho o v)$ gibt er die folgenden drei Beispiele mit den drei Artikeln $\dot{o}, \dot{\eta}$ und $\tau o ́$

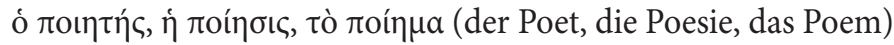

\footnotetext{
2 Diese Bindung von Genus an „Kongruenz“ war auch in der modernen Sprachwissenschaft schon vor Hockett üblich: So taucht der Genusbegriff in L. Bloomfields „Language“ - zu dessen 1984er Auflage Hockett ein Vorwort beigesteuert hat - nicht zufällig gerade im Kongruenzkapitel (12.7) auf: Die Artikel, so Bloomfield mit Bezug auf die Substantive des Deutschen, stünden „in agreement with the so-called gender-classes, into which German nouns are divided. These genders are arbitrary classes, each of which demands different congruent forms in certain kinds of accompanying words." (1933: 192) Und etwas mehr als 50 Jahre vorher schreibt H. Paul, den Bloomfield gut gekannt hat:

„Das sprachliche Mittel, woran wir jetzt das grammatische Geschlecht eines Substantivums erkennen, ist die Kongruenz, in welcher mit demselben einerseits Attribut und Prädikat, andererseits ein stellvertretendes Pronomen steht." (1966: 264)

3 Ich zitiere und übersetze den griechischen Text nach der von G. Uhlig herausgegebenen Ausgabe „Grammatici Graeci I,1“.
} 
Richtig interessant aber wird es erst, wenn wir das Zitat fortsetzen:

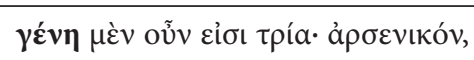

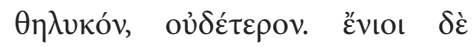

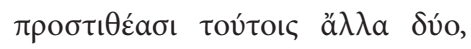

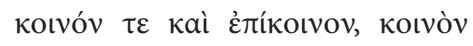

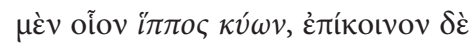
oĩov $\chi \varepsilon \lambda_{l} \delta \dot{\omega} v \dot{\alpha} \varepsilon \tau \tau^{\circ} \varphi^{4}$
Genera gibt es drei: Maskulin, feminin, neutrum. Manche aber fügen diesen noch zwei weitere hinzu, koinón und

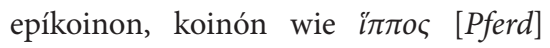
$\kappa u ́ \omega v[H u n d]$ und epíkoinon wie $\chi \varepsilon \lambda \iota \delta \omega ́ v$ [Schwalbe] ảztós [Adler].

Dionysios weist also darauf hin, daß neben den drei bekannten Genera noch andere unterschieden werden, und er exemplifiziert das $\gamma \dot{\varepsilon} v 0 \varsigma$ кoเvóv (= gemeinsames

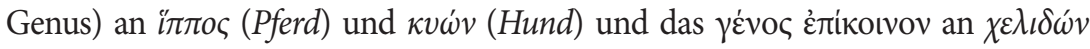
(Schwalbe) und ả\&tós (Adler). Bei dem génos koinón, um uns hier auf dieses Genus zu konzentrieren, haben wir ein und dasselbe Substantiv, also etwa auch $\beta$ ov $\varsigma$ (Rind) oder $\dot{\alpha} \lambda \varepsilon \kappa \tau \rho v \omega \dot{v}(H u h n)$, und je nach dem Geschlecht wechseln die Artikel, ohne daß das Substantiv sich (und das heißt: seine Deklinationsklasse) änderte:

maskulin

$$
\begin{aligned}
& \text { ó ï } \pi \pi \text { oৎ (der Hengst) }
\end{aligned}
$$

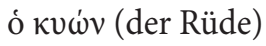

\section{feminin}

$$
\begin{aligned}
& \text { ๆ }
\end{aligned}
$$

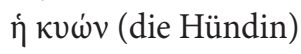

Das $\gamma$ ćvoc Koเvóv, dieses „gemeinsame Genus", ist deshalb ein 4. Genus, weil sich bei diesen Substantiven die Artikel anders verhalten als bei dem maskulinen, dem femininen und dem neutrischen Genus. Das heißt nicht, daß sich das 4. Genus durch einen 4. Artikel auszeichnet. Auch bei i $\pi \pi \circ$ s et al. haben wir ja keine anderen Artikel als die bekannten. Der Punkt ist: Bei ï $\pi$ to et al. kann ich nicht nur 1 der drei Artikel verwenden, sondern zwei, und das macht einen klaren Unterschied.

Die (nomen-externen) Artikel entscheiden also auch bei gegenteiliger (nomen-interner) Deklinationsklasse über das Genus des Nomens: Maskulin ist,

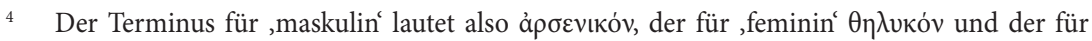

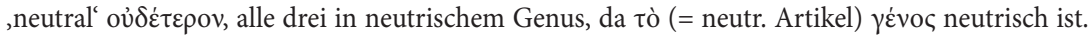
Lange vor Dionysios Thrax hat schon Aristoteles (384-322 v. Chr.) die Genera ,männlich', ,weiblich' und ,dazwischen ( $\mu \varepsilon \tau \alpha \xi u ́)$ unterschieden (vgl. Arens 1969: 15, Brandenburg 2005: 136).

5 Arens (1969: 23) übersetzt $\gamma \varepsilon$ ćvoৎ Koเvóv mit „Doppelgeschlecht“.
} 
wo der maskuline Artikel $\delta$ voransteht, feminin, wo der feminine Artikel $\dot{\eta}$ vorangeht, und neutrum, was mit dem neutrischen $\tau o ́$ steht, und $\gamma \dot{\varepsilon} v 0 \varsigma$ Koเvóv liegt vor, wenn je nach Sexus sowohl $\delta$ als auch $\dot{\eta}$ stehen kann. ${ }^{6}$

\section{Genuscluster, Kerne und Satelliten: Ein Blick ins Lateinische}

In dem artikellosen Lateinischen sind die Pronomina und viele Adjektive ebenso dreifach genusdifferenziert wie die Substantive:

$$
\begin{aligned}
& \text { ille dominus bonus (jener gute Herr) } \\
& \text { illa femina bona (jene gute Frau) } \\
& \text { illud vinum bonum (jener gute Wein) }
\end{aligned}
$$

Das Substantiv, (der Nukleus) hat in seinem lateinischen Gravitationsfeld also Genus-Satelliten in unterschiedlichen Stellungen und Entfernungen und bildet zusammen mit seinen Satelliten ein, sagen wir: Genuscluster:

\begin{tabular}{|c|c|c|c|c|c|c|}
\hline Nukleus & \multicolumn{6}{|c|}{ S t e l 1 i t e n } \\
\hline Subst. & Possess. & Adj. & Relativ. & Personal. & $\begin{array}{c}\text { Nahde- } \\
\text { monstrativ. }\end{array}$ & $\begin{array}{c}\text { Ferndemon- } \\
\text { strativ. }\end{array}$ \\
\hline hortus & suus & bonus & qui & is & hic & ille \\
\hline casa & sua & bona & quae & ea & haec & illa \\
\hline vinum & suum & bonum & quod & id & hoc & illud \\
\hline
\end{tabular}

Tab. 1: Lateinisches Genuscluster

Auch im Lateinischen kommt es vor, daß sich zwei Substantive zwar formal ganz gleich verhalten, d.h. dieselben Endungen aufweisen (und damit zur gleichen „Deklinationsklasse“ gehören), ihre Satelliten sich aber unterschiedlich verhalten. Da gibt es einmal Fälle wie agricola (Bauer) oder poeta (Dichter), die exakt so dekliniert werden wie femina, doch die maskulinen Satelliten zeigen, daß sie maskulin sind:

\begin{tabular}{|l|l|l|l|l|l|}
\hline agricola & bonus & qui & is & hic & ille \\
\hline
\end{tabular}

\footnotetext{
6 Die Genera werden also, so Brandenburg (2005: 579), „,aus der Syntax und Kongruenz [griech. б $\mu \varphi \omega v i$ ia, P.C.] mit den Artikeln“ erkannt, die den verschiedenen Nomina „vorangestellt werden“.
} 
Das Substantiv agricola, der Nukleus, fordert also maskuline Satelliten, zeigt aber sein Genus selbst nicht; das tun nur die Satelliten.

Und dann gibt es daneben etwa jene Substantive, bei denen wir wie bei dem Fall des génos koinón von $\delta / \grave{\eta} i \pi \pi o \varsigma$ (der Hengst / die Stute) ein gemeinsames Genus („genus commune“) haben: ${ }^{7}$

\section{maskulin}

ille bos (jener Stier)
ille sacerdos (jener Priester) feminin

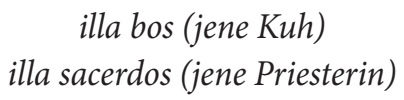

Das maskuline bzw. feminine Genus zeigen auch hier nicht die Nuklei, sondern die Satelliten ille bzw. illa. Wir können also wieder sagen: Es ist maskulin, wo die Satelliten maskulin sind, feminin, wo sie feminin sind, und neutrum, wo sie neutrisch sind.

Wie man sieht, ist Genus eine Flexionskategorie der Satelliten, nicht aber eine des Nukleus. Genus ist eine nominale Kategorie, wenn man mit ,nominal' die Artikel, Adjektive und Pronomina meint. Genusverschiedene Wortformen gibt es nur bei den Satelliten (bonus/bona/bonum etc.), beim Substantiv nicht (femina). Deshalb „kongruieren“ der Nukleus und seine Satelliten zwar in den Wortformkategorien Kasus und Numerus, aber nicht in der Wortkategorie Genus. Das genusfeste Wort femina fordert von dem genusflektierenden Satelliten bonus/bona/bonum die Wortform bona. Also liegt, anders als bei den Kongruenzkategorien Kasus und Numerus, beim Genus eine Form von Rektion vor.

Anders als das deutsche „convergent system“ (Corbett 1991: 155), das im Plural ja keine Satellitengenera ${ }^{8}$ kennt, reflektieren die griechischen und lateinischen Satelliten im Plural dieselben Genera wie im Singular. ${ }^{9}$ Die Wortkategorie Genus betrifft dort also tatsächlich alle Wortformen.

\footnotetext{
7 Dazu gehört neben infans (Kind) auch parens, von dem das ebenfalls maskuline und feminine englische parent stammt, von dem viele wie bei dem lateinischen parentes (Eltern) nur den Plural parents kennen.

8 Zu einer anderen als der ja auch von mir hier vertretenen satellitenorientierten Genusbestimmung à la Hockett vgl. V. Ágel 2005.

9 So verrät auch Mozarts italienisches Cosi fan tutte anders als das deutsche So machen es alle, daß mit tutte nur die Frauen gemeint sind (tutte le donne).
} 


\section{Genus im Englischen}

Im heutigen Englisch gibt es innerhalb solcher Nominalphrasen wie the nice man / the nice woman / the nice book bekanntlich keine Genusunterschiede. Bei den Personal-, Reflexiv- und Possessivpronomina gibt es die üblicherweise als solche interpretierte Genusunterscheidung von he/she/it bzw. himself/herself/ itself bzw. his/her/its:

$\begin{array}{llll}\text { a/the nice man } & \text { himself } & \text { he } & \text { his } \\ \text { a/the nice woman } & \text { herself } & \text { she } & \text { her } \\ \text { a/the nice book } & \text { itself } & \text { it } & \text { its }\end{array}$

Nehmen wir nun die Relativa hinzu, sehen wir zuerst einmal, daß es nicht mehr, wie bisher, drei, sondern (abgesehen von that, das für who und which stehen kann) nur noch zwei Pronomina gibt, who und which:

\begin{tabular}{|c|c|c|c|c|c|}
\hline Artikel & Substantiv & Relativ. & Reflexiv. & Personal. & Possessiv. \\
\hline a/the & man & who & himself & he & his \\
\hline a/the & woman & who & herself & she & her \\
\hline a/the & book & which & itself & it & its \\
\hline
\end{tabular}

Tab. 2: Satellitengenera bei den englischen Pronomina

Die im Personal-, Possessiv- und Reflexivpronomen unterschiedenen drei Genera fallen also beim Relativum in zweien zusammen. Während sich die Personalpronomina (und genauso die Possessiva und Reflexiva) nach dem sog. „natürlichen Geschlecht" richten, richten sich who und which danach, ob das Bezeichnete eine Person ist oder nicht. Es gibt keinen Grund, den durch who und which gemachten Unterschied anders zu interpretieren als den durch he, she und it etc. gemachten und das heißt: anders als einen Unterschied im Genus.

R. Quirk, / S. Greenbaum, / G. Leech / J. Svartvik (1991) unterscheiden zwischen dem Genus englischer Nomina und dem der Personal- und Reflexivpronomina he/himself und she/herself: Letztere zeigen „offen“ das Genus ,maskulin bzw. ,feminin'; anders die Nomina: „Gender in English nouns may be described 
as ,notional' in contrast to the ,grammatical' or ,overt' gender of nouns in languages such as French, German, or Russian (...).“ (ebd. 314)

Die Nomina werden im Englischen also nicht „grammatically“ wie etwa im Deutschen, sondern „semantically“ gruppiert, und zwar ,according to their coreferential relation with personal, reflexive and wh-pronouns" (ebd.); auch hier also wieder der deutliche Rekurs auf die „associated words“. Diese verdeckten („covert“) Genera der Nomina nennen Quirk et al. denn auch nicht ,maskulin und ,feminin', sondern ,male und ,female. Aber halten wir fest: Sie sprechen von "gender“.

Auf der Basis der Koreferenzbeziehungen ergeben sich „nine gender classes“ (ebd.), die aus 1 unbelebten („inanimate“) Genusklasse und 8 belebten („animate") Klassen bestehen. Die 8 belebten bestehen ihrerseits aus 4 personalen Genusklassen (male, female, dual, common) und 4 nonpersonalen (Kollektiva, höhere männl. Tiere, höhere weibl. Tiere, niedere Tiere). Hier zur Veranschaulichung die 4 personalen:

\begin{tabular}{lll}
$\begin{array}{l}\text { gender } \\
\text { class }\end{array}$ & Beispiel & \multicolumn{1}{c}{$\begin{array}{l}\text { koreferentes } \\
\text { Pronomen }\end{array}$} \\
male & $\begin{array}{l}\text { brother } \\
\text { sister } \\
\text { female } \\
\text { dual } \\
\text { doctor }\end{array}$ & $\begin{array}{l}\text { who-he } \\
\text { who-she } \\
\text { who-he/she } \\
\text { baby }\end{array}$ \\
who-he/she/it
\end{tabular}

Tab 3: Vier personale Genusklassen im Englischen

Diese Genusklassen sind ,personal', weil ihr Relativpronomen who ist:

\section{Relativpronomen}

personal gender nonpersonal gender who

which 
Unterscheiden tun sich die männliche und die weibliche Genusklasse in den Personalpronomina he und she. Das doctor gehört zur Klasse ,dual', weil he und she stehen können, und baby gehört zur Klasse ,common', weil das Pronomen he, she und it sein kann. Und was sehen wir hier? Das Prinzip ist genau dasselbe wie schon bei Dionysios Thrax: Auch bei ihm ergab sich das $\gamma \dot{v} v o \varsigma$ кoเvóv, das "gemeinsame Genus", daraus, daß man mehr als nur den üblichen 1 Satelliten (Artikel) verwenden konnte.

Die „Gender distinctions in pronouns“ (ebd. 341) sehen dann so aus:

\section{PERSONAL GENDER}

$$
\text { masculine he him his himself }
$$

feminine sheher her(s) herself

NONPERSONAL GENDER it its itself ${ }^{10}$

Tab. 4: Pronominale Genera im Englischen

Einen „masculine/feminine contrast“ (ebd.) gibt es nur bei den in Tab. 4 angeführten Pronomina, den „personal/nonpersonal contrast“ gibt es auch bei den Relativ- und Indefinitpronomina (who - which, somebody - something). Die Wahl zwischen den „masculine and feminine pronouns“ (ebd. 342) basiert - so die Autoren unter der Überschrift „Masculine and feminine gender“ - primär auf dem Sexus. ${ }^{11}$

10 Sehr klar formuliert L. Bloomfield, wie die Pronomina Genera konstituieren: „Our selection of substitutes, then, divides English substantive expressions into the sub-classes of personal (replaced by who and he-she) and non-personal (replaced by what and it), and it subdivides the personal singulars into the sub-classes of male (replaced by he) and female (replaced by she)." (1933: 248)

Und etwas später schreibt er, die maskulinen Nomina seien „defined as those for which the definite substitute is $h e^{\text {“, }}$ und die femininen seien ,similarly defined by the use of the substitute she (ebd. 253).

11 Bei dem „agreement“ zwischen Pronomina und Antezedenten handele es sich zwar eher um Koreferenz als um „grammatical concord“, aber es sei angemessen, das Phänomen unter der Überschrift „Concord“ zu behandeln (ebd. 768): „Thus the relative pronouns who, whom, and which agree with their antecedent in the superordinate clause in gender, the first two being personal, and the last nonpersonal [...]. Personal and possessive pronouns [...] in the 3rdperson singular (he, she, it) also agree with their antecedents in gender [...].“( ebd. 769) 
Corbett klassifiziert auch Sprachen wie das Englische als Genussprachen, und er hält es auch für kein Gegenargument gegen die Interpretation einer Sprache „as having a gender system" (1991: 112), wenn sich das nur in den Pronomina zeigt („if this is reflected only in the pronoun“; ebd.). Da dieser Ansatz nicht universell akzeptiert sei, nennt er solche Systeme auch „pronominal gender systems“ (ebd. 5).

\section{Und schließlich: Das Ungarische}

Noch einmal zur Erinnerung Hockett: „Genders are classes of nouns reflected in the behavior of associated words.“ (1958: 231) Gibt es auch im Ungarischen Substantivklassen, „classes of nouns“, deren Unterschiede sich im Verhalten von assoziierten Wörtern, „in the behavior of associated words“, widerspiegeln?

Solche Korrespondenzen („Kongruenz“) gibt es in der Tat auch im Ungarischen, und auch dort sind es die Pronomina, die jene „associated words“ darstellen. Dort haben wir sogar gleich drei Pronominapaare:

- Erstens das aus dem Personalpronomen ő und den Demonstrativa ez und $a z$ bestehende Paar,

- zweitens das aus den Relativa aki und ami bestehende Paar und

- drittens das aus den (aki und ami formal entsprechenden) Fragepronomina $k i$ und $m i$ bestehende Paar. ${ }^{12}$

Wenn man den Aussagen der Muttersprachler glauben darf, richten sich diese „associated words“ klar nach semantischen Kriterien:

$$
\begin{array}{llll}
\text { ״ } & \rightarrow \text { Menschen } & \mathrm{ez} / a z & \rightarrow \text { Dinge } \\
a k i & \rightarrow \text { Menschen } & a m i & \rightarrow \text { Dinge } \\
k i & \rightarrow \text { Menschen } & m i & \rightarrow \text { Dinge }
\end{array}
$$

Die drei Pronominapaare erlauben auf den ersten Blick also die folgenden Cluster, in denen Nukleus-Substantive mit verschiedenen Pronominalsatelliten korrespondieren, und zwar im Singular und im Plural:

12 Dieses - $k i$ und - $m i$ finden wir auch z.B. in valaki (jemand) und valami (etwas). 


\begin{tabular}{|c|c|c|c|}
\hline Substantiv & $\begin{array}{c}\text { Relativ- } \\
\text { pronomen }\end{array}$ & $\begin{array}{c}\text { anaphorisches/deikti- } \\
\text { sches Pronomen }\end{array}$ & $\begin{array}{c}\text { Frage- } \\
\text { pronomen }\end{array}$ \\
\hline férf (Mann) & aki & ö & ki \\
\hline nő (Frau) & aki & ö & ki \\
\hline könyv (Buch) & ami & ez/az & $\mathrm{mi}$ \\
\hline
\end{tabular}

Tab. 5: Zwei Reihen von Pronominalsatelliten im Ungarischen

Diese scheinbar so klare Gegenüberstellung

ö/aki/ki $\rightarrow$ Menschen ez/az/ami/mi $\rightarrow$ Dinge

ist indes problematisch, und das aus mehreren Gründen, und wir konzentrieren uns im Folgenden auf ö / aki vs ez/az / ami:

- Die Gegenüberstellung von Menschen und Dingen ist nicht exhaustiv: Es gibt noch anderes, über das man reden kann, z.B. Tiere.

- Bei genauerem Hinsehen entsprechen sich ő und aki funktional gar nicht in dem suggerierten Maße (und ez/az und ami ebenfalls nicht).

Das Problem der mangelnden Exhaustivität hat T. Forgács nicht, wenn er, unter Hinzunahme von amely, zu aki/ami schreibt:

Aki (manchmal nur ki) ,wer; der/die/das; welcher/-e/-es' bezieht sich immer auf Menschen [...].

Sonst werden amely und ami (ganz selten auch $m i$ ) verwendet. (Forgács 2004: $\$ 216$; Hervorhebung i.O. $)^{13}$

Und auch B. Keszler / K. Lengyel haben dieses Problem nicht, wenn sie, ebenfalls unter Hinzunahme von amely, zum selben Thema schreiben:

aki ,der/die/das [belebt]; ami ,der/die/das [unbelebt]'; [...] amely,der/ die/das [unbelebt]' (Keszler / Lengyel 2008: 65)

\footnotetext{
13 Ähnlich I. Tótfalusi (1997: 60): Sei von Personen die Rede, verwende man aki, sonst amely („ha személyről van szó, aki dukál, más esetben amely kell“).
} 
Doch noch offensichtlicher als daß sie kein Exhaustivitätsproblem haben ist, daß die Unterscheidungen, Menschen - Sonstiges' und ,belebt - unbelebt' nicht übereinstimmen. Das Belebtheitskriterium spricht aki ja einen viel größeren Umfang zu als die Behauptung, aki beziehe sich „immer auf Menschen“. Daß letzteres empirisch auch falsch ist, zeigt etwa das Beispiel der Tiere: Wenn sich aki „immer auf Menschen“ bezöge, müssten alle Tiere in den ami-Bereich fallen, und das tun zumindest die Haustiere sicher nicht:

$$
\text { A } 9 \text { éves kutyám, akivel ... (Mein 9jähriger Hund, mit dem ...) })^{14}
$$

Aber auch das Kriterium ,belebt - unbelebt' ist nicht völlig unproblematisch: Abgesehen etwa von den Nicht-Haustieren stellt sich die Frage: Wie steht es z.B. mit den Toten? Die gehören, obwohl unbelebt, nicht zum ami-Bereich: Bartók Béla, aki ( ${ }^{\star}$ ami $) . .{ }^{15}$

Noch weniger eindeutig sind die Grenzen zwischen ő und ez/az. Sowohl die Regel, wonach $e z$ und $a z$ Gegenstände (und keine Menschen) bezeichnen, als auch die Regel, wonach ő Personen (und keine Gegenstände) bezeichnet, ist in bestimmten Fällen aufgehoben. In den zwei Beispielen

(2) A költőnek van egy új barátja. Az minden nap ír neki egy levelet. (Der Dichter hat einen neuen Freund. Der schreibt ihm jeden Tag einen Brief.)

(3) Az a szép, az a szép, akinek a szeme kék. ${ }^{16}$

(Der/die ist schön, der/die ist schön, der/die blaue Augen hat.)

\footnotetext{
$14 \quad$ Noch einmal Tótfalusi (1997: 60): Bei Ausdrücken wie család (Familie) oder csapat (Mannschaft) sei zwar von Personen die Rede, aber da die Ausdrücke selbst keine Personen bezeichneten, müsse (!) es amely heißen. Die Frage „Wie heißt die Familie, die unter uns wohnt?" beantworteten alle von mir befragten MuttersprachlerInnen mit ... a család, aki ... Im Falle von die Mannschaft, die gewonnen hat antworteten sie z.T. mit amely, z.T. mit aki, z.T. mit ami-

15 Ich übersetze einen Dialog zwischen mir und einer Muttersprachlerin:

Sie: „Aki verwenden wir bei Personen.“

Ich: „Heißt es a kutyám, aki oder ami?"

Sie: „A kutyám, aki, denn der Hund ist ein Lebewesen.“

Ich: „Heißt es Bartók Béla, aki oder ami?“

Sie: „Bartók Béla, aki, denn Bartók ist eine Person.“

16 In Fn. 1 habe ich auf zwei meiner Kontakte mit Péter Bassola hingewiesen. Ich möchte an dieser Stelle nicht verschweigen, daß ich bei einer dritten Gelegenheit (in einem Weinkeller in Villány) auch das obige Lied von ihm gelernt habe.
} 
bezieht sich $a z$ auf Menschen: Im ersten Fall macht das $a z$ wie auch das der den Bezug auf den Freund klar. Im zweiten Fall ist das az wie das der/die kataphorisch und auch in dieser Funktion klar die erste Wahl.

In dem folgenden Beispiel bezieht sich ő auf einen Gegenstand:

(4) Szene: Im Elektromarkt. Ich stehe mit einem Verkäufer vor einer Waschmaschine. Ich (mit Bezug auf die Waschmaschine, über die wir gerade geredet haben): „Akkor kérem.“ (Die möchte ich dann.)

Verkäufer: „Öt?“ (Sie?)

Ich (scherzhaft): „Nem őt, hanem azt!“ (Nicht sie, sondern die.)

Er (lacht): „Igaza van. Hivatalosan azt, de én szoktam őt is mondani.“ (Sie haben Recht. Eigentlich heißt es $a z$, aber ich sage auch öt.)

Ich: „Miért? Mi a különbség az az és ő között?“ (Warum? Was ist der Unterschied zwischen dem $a z$ und $o$ ?)

Er: „Az ő megszemélyesítés.“ (Das ő ist eine Personifizierung.)

Natürlich gilt ein solches Beispiel einerseits als „Ausnahme“, andererseits passiert diese Personifizierung häufig. Ich habe erlebt, daß sich Sprecher auf einen Rasenmäher, mein Auto, ein Schlüsselbund, einen Becher Kaffee oder einen Ort auf einer Karte (Ő Nemesvámos!) mit ő bezogen haben. Wenn jener Verkäufer ${ }^{17}$ mit seiner Diagnose „megszemélyesítés“ recht hat, dann zeigt das: ő bezeichnet primär Individuen, d.h. Personen, Tiere oder auch Gegenstände, die dem Sprecher und/oder Hörer vertraut sind, das ez/az-ami-Paar bezeichnet primär anderes.

$\mathrm{Daß}$ sie in Wirklichkeit nicht so konform sind, wie wir suggeriert haben, das gilt auch für die englischen Pronomina. So wird man sich etwa auf seinen geliebten Hund nicht nur mit he/she, sondern auch mit who (und nicht mit which) beziehen. Corbett (1991: 12) nennt zwei Beispiele, in denen sich ein maskulines und ein feminines Personalpronomen und ein feminines Possessivum „regelwidrig“ auf Nichtpersonales beziehen:

(5) (Kundin mit Bezug auf einen Bettbezug:) Is he washable?

(Surfer mit Bezug auf eine Welle:) Catch her at her height. (kursiv i. O.)

17 Ich habe den Eindruck, daß insbesondere Verkäufer diese megszemélyesítés lieben, und diesen Eindruck hat mir eine Vertreterin dieser Zunft auch bestätigt. 
Ein anderer Fall als diese Personifizierungen liegt vor im Falle der englischen „boat nouns" und ihrer deutschen Verwandten. Die sind ja deshalb berühmt, weil sie „feminin“ sind:

(6) The Titanic was on her maidentour. She ...

Die Titanic war auf ihrer Jungfernfahrt. Sie ... ${ }^{18}$

Auch hier beziehen sich das feminine Personal- und Possessivpronomen she und her ja eigentlich ebenfalls „regelwidrig“ auf Nichtpersonales. Das englische Relativpronomen, und deshalb ist dieser Fall für uns überhaupt von Interesse, macht diese Femininisierung nun aber nicht mit:

(7) The Titanic, which ( ${ }^{*}$ who) ... her ... she ...

Das Relativum which durchbricht diese Regelwidrigkeit der personal gender-Pronomina, indem es sich an die „eigentlich“ geltenden Regeln hält. Im Ungarischen beobachten wir Ähnliches: Der Sprecher kann sich auf sein altes Motorrad, für ihn ein Individuum wie etwa auch sein Hund, nicht nur mit öreg (alt für Personen und Tiere), sondern durchaus auch mit dem personifizierenden ő (ővel, kurz vele $=$ mit ihm) beziehen:

(8) Ott állt az öreg motorja is. Vele minden nyáron ide-oda utazott Olaszországban. (Dort stand auch sein altes Motorrad. Mit ihm war er jeden Sommer in Italien herumgereist.)

Aber bei dem Relativpronomen ist Schluss damit: ${ }^{19}$

\footnotetext{
18 Hier werden nicht individuelle Gegenstände personifiziert, sondern es wird eine ganze Klasse gekennzeichnet. Wenn ich von meinem Motorrad als die Honda spreche, personifiziere ich es auch nicht, sondern benutze lediglich, wie es auch etwa die Franzosen tun, wenn sie bei la Volkswagen das feminine Genus von (la) voiture verwenden, das Genus eines Leitnomens wie Maschine. Daß wir bei Schiffen feminine Satelliten verwenden (die Gorch Fock ... sie), verdankt sich aber wohl keinem Leitnomen, sondern vermutlich eher dem Englischen.

19 Dieses unterschiedliche Verhalten des englischen und ungarischen Relativums läßt sich vermutlich wie der deutliche Fall etwa der Unmöglichkeit von ein/das Mädchen, *die bei gleichzeitig im Falle einer gewissen textuellen Distanz gut möglichem ein/das Mädchen ... sie ... ihr durch textuelle Nähe erklären.
} 
(9) Ott állt az öreg motorja is, amivel (*akivel) minden nyáron ide-oda utazott Olaszországban. (Dort stand auch sein altes Motorrad, mit dem er jeden Sommer ...)

Die Verteilung von aki vs ami ist also ganz deutlich viel strenger als die von ő vs ez/az. Auf Unbelebtes kann man sich u. U. zwar gut mit dem Personalpronomen beziehen, nicht aber mit aki.

Zur Erinnerung: Die personalen und die nonpersonalen Genusklassen des Englischen unterschieden sich durch who und which:

\section{Relativpronomen}

\section{personal gender nonpersonal gender}

who
which

Wird durch die Unterscheidung von ő und $e z / a z$ und insbesondere die weniger personifizierungsanfällige strengere und damit sozusagen wasserdichte Unterscheidung von aki und ami auch im Ungarischen so etwas wie ein „pronominal gender system" konstituiert?

\section{Relativpronomen}

\section{personales Genus nichtpersonales Genus}

$a k i$

$a m i$

Ich schließe mich bei der Beantwortung dieser Frage jener eindeutigen Position an, die von Corbett (1991) und 67 Jahre vorher von Otto Jespersen (1924) folgendermaßen formuliert wird:

Since agreement is taken as the criterion for gender, there are no grounds for drawing a distinction between languages in which nouns are divided into groups according to sex, and those where human/non-human or animate/non-animate are the criteria. (Corbett 1991: 5)

By the term gender is here meant any grammatical class-division (...), whether the division be based on the natural division into the two sexes, or on that between animate and inanimate, or on something else. (Jespersen 1924: 55) 
Und um damit noch einmal im doppelten Sinne auf „unsere Anfänge“ zurückzukommen: Wir haben das personale aki- und das nonpersonale ami-Genus des Ungarischen nach demselben Kongruenz-Prinzip eruiert, mit dem schon Dionysios Thrax seine 3 bzw. 5 Genera eruiert hat. Er hat naheliegenderweise als „associated words“ die Artikel verwendet, ganz besonders deutlich im Falle des génos koinón à la

\section{maskulin}

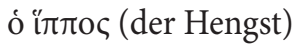

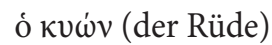

\section{feminin}

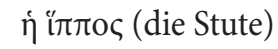

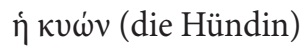

Die artikellose lateinische Grammatik konnte das nicht, hatte aber die von uns beobachteten Genuscluster. Wie die Genera gerade bei dem genus commune à la

maskulin

ille bos (jener Stier)

ille sacerdos (jener Priester)

\section{feminin}

illa bos (jene Kuh)

illa sacerdos (jene Priesterin)

und bei den Substantiven des Typs agricola

\begin{tabular}{|c|c|c|c|c|c|}
\hline Subst. & Adj. & Relativ. & Personal. & $\begin{array}{c}\text { Nahdemons- } \\
\text { trativa }\end{array}$ & $\begin{array}{c}\text { Ferndemons- } \\
\text { trativa }\end{array}$ \\
\hline agricola & bonus & qui & is & hic & ille \\
\hline
\end{tabular}

Tab. 6: Genusbestimmung durch Satelliten

ganz unabhängig von den Substantiven durch "the behavior of associated words“, hier: Artikel, Adjektive und Pronomina reflektiert werden, so werden auch die ungarischen Genera durch „associated words“ reflektiert, und zwar auch hier durch Pronomina, allen voran die Relativa. 


\section{Postscriptum}

Als ich mit der ersten Version dieses Textes lange fertig war, bin ich auf eine Stelle in B. Collinders „Comparative Grammar of the Uralic Languages“ (1960) gestoßen. Dort gibt es etwas mehr als eine Seite zu „Gender and Sex“, und da finden sich folgende Aussagen:

In Selkup, some of the cases of the nouns have two different case endings: one for designations of living beings, the other for all the rest. This constitutes, then, two genders: genus animatum and genus inanimum [sic!]. [...]

In the Uralic languages, with the exception of Selkup, there is no grammatical gender-category. But a lexical distinction between genus humanum and genus non humanum exists insofar as there are different "personal" and non-personal pronouns, including interrogative (and relative) pronouns, e.g. Hungarian $k i$, who, mi, what. (Collinder 1960: 241f.) ${ }^{20}$

C. I. Beckwith kennt Collinders Hinweis auf das ki/mi-Paar und dessen Interpretation im Sinne von genus humanum vs. genus non humanum. ${ }^{21}$ Er beginnt seinen Aufsatz „Classifiers in Hungarian“ dennoch mit der von uns zu Beginn zitierten Aussage, wonach „Uralic languages, in particular Hungarian, do not have gender" (1992: 197). Zu den einfachsten und verbreitetsten Typen sprachlicher Klassifikation („taxonomy“) gehöre eine Unterscheidung vom Typ ,belebt - unbelebt', ,menschlich - nicht menschlich oder , männlich - weiblich ' wie z.B. die „overtly concordial gender systems“ (198) des Lateinischen, Russischen und Arabischen. Wenn man, so Beckwith, die gerade angesprochene Unterscheidung wie im Falle des ungarischen Paares ki (wer?) vs mi (was?) bis zur Ebene der Fragepronomina ${ }^{22}$ bringe, sei es wahrscheinlich, „that every human

\footnotetext{
20 In Collinder (1965) gibt es auf S. 57 eine gute halbe Seite zu „Gender and Sex“, und da finden sich die obigen Aussagen ebenfalls.

21 Er erwähnt die entsprechende Stelle in Collinder (1965) bereits im zweiten Satz seines Aufsatzes.

22 Wie Collinder nennt auch Beckwith nur das ki/mi-Paar. Collinder erwähnt die Relativa, allerdings ohne Bezug speziell aufs Ungarische und nur in Klammern.
} 
language exhibits taxonomic phenomena, indicating that the subject deserves far more attention than it has received to date" (ebd.). ${ }^{23}$

\section{Literatur}

Ágel, Vilmos (2005): Das fünfte und sechste Genus - und die anderen. In: Fuhrhop, Nanna (Hrsg.): Virtuelle Festschrift für Peter Eisenberg. Beckwith, Christopher I. (1992): Classifiers in Hungarian. In: Kenesei, István / Pléh, Csaba (Hrsg.): The Structure of Hungarian. Szeged: JATE, 197-206. Bloomfield, Leonard (1933): Language. New York: Holt \& Co. Brandenburg, Philipp (2005): Apollonios Dyskolos. Über das Pronomen. Einführung, Text, Übersetzung und Erläuterungen. München, Leipzig: K.G. Saur.

Collinder, Björn (1960): Comparative Grammar of the Uralic Languages. Stockholm: Almqvist \& Wiksell.

23 Nicht behandelt habe ich hier zwei andere nicht nur im Ungarischen beobachtbare Formen von Substantivklassifikation, nämlich erstens die an die deutschen Korrespondenzen vom Typ -heit/keit ... die erinnernde explizite Korrespondenz ,Person' ausdrückender Suffixe wie etwa -ista, -s, -ász/-ész mit ö und aki und von Suffixen wie etwa -ás oder -de/-da mit ez/az und ami:

$\begin{array}{ll}\text { egyetemista (Student), alkoholista } & \text { aki ...ő } \\ \text { órás/iskolás (Uhrmacher/Schüler) } & a k i \ldots o ̋ \\ \text { halász/kertész (Gärtner/Fischer) } & a k i \ldots o ̋ \\ \text { olvasás/írás (Lesen/Schreiben) } & \text { ami ...e ez/az } \\ \text { sörfözde/óvoda (Brauerei/Kindergarten) ami ...e ez/az }\end{array}$

Und zweitens nicht behandelt habe ich die ungarischen „classifiers“, die ja, wie der Name schon sagt, ebenfalls Substantive klassifizieren. Beckwith (1992: 201) nennt 6 „classifiers“ mit Beispielen (ich übersetze):

fó (Kopf): három fö gyalogos (3 Kopf Fußgänger)

fej (Kopf): négy fej hagyma/káposzta/saláta (4 Kopf Zwiebeln/Kohl/Salat)

szem (Auge): hét szem szölö/szilva/búza (7 Auge Wein/Pflaumen/Weizen)

tö (Stock): egy tö szölö (1 Stock Trauben)

szál (Faden): $\quad$ hét szál virág/szalma/16 szál gyertya/néhány moszatszerü szál haj (7 Faden Blumen/Stroh/16 Faden Kerzen/einige algenartige Faden Haare)

kötet (Band): három kötet könyv (3 Band Bücher)

Beides sind wohl zweifellos Formen der Klassifikation von Substantiven, und im ersteren Fall liegt ebenso zweifellos sogar explizite Genuskorrespondenz vor. Nicht behandelt habe ich sie, weil sie nur einen relativ kleinen Ausschnitt der Substantive betreffen und so nicht zu Genera führen. 
Collinder, Björn (1965): An Introduction to the Uralic Languages. Berkeley: University of California Press.

Corbett, Greville (1991): Gender. Cambridge: Cambridge University Press.

Forgács, Tamás (2004): Ungarische Grammatik. 2., verb., Aufl. Wien: Edition Praesens.

Grammatici Graeci I, 1: Dionysii Tracis Ars Grammatica. 1883 herausgegeben von Gustav Uhlig. Leipzig: Teubner.

Jespersen, Otto (1924): The Philosophy of Grammar. London: Allen \& Unwin.

Keszler, Borbála (Hrsg.) (2001): Magyar grammatika. Budapest: Nemzeti Tankönyvkiadó.

Keszler, Borbála / Lengyel, Klára (2008): Ungarische Grammatik. Hamburg: Buske.

Paul, Hermann (1966): Prinzipien der Sprachgeschichte. 7. Aufl. Tübingen: Niemeyer.

Quirk, Randolph / Greenbaum, Sidney / Leech, Geoffrey / Svartvik, Jan (1991): Comprehensive Grammar of the English Language. 9. Aufl. London: Longman.

Tompa, József (1985): Kleine ungarische Grammatik. Budapest: Akadémiai Kiadó.

Tótfalusi, István (1997): Magyar nyelvhelyességi kéziszótár A-tól Z-ig. Budapest: Merényi Könyvkiadó. 